\title{
Modelo de simulação de sistemas de pastejo rotativo e contínuo de azevém (Lolium multiflorum lam.) na bovinocultura
}

\author{
Simulation model of rotative and continuous grazing systems of ryegrass \\ (Lolium multiflorum Lam.) in bovine cattle
}

Benedito Silva Neto $^{1}$ Mariane Schneider ${ }^{2}$ Julio Viegas $^{3}$

\section{RESUMO}

O principal objetivo do trabalho é propor um modelo de simulação de sistemas dinâmicos para a análise do manejo de pastagens visando a maximizar o consumo de forragem pelos animais. A forrageira utilizada foi o azevém anual, pastado por bovinos adultos. Foram consideradas diversas variáveis como: lotação animal, taxa de crescimento do pasto, taxa de apreensão da forragem pelos animais, primeira entrada dos animais na pastagem após a emergência (início do pastejo) e ciclo de rotação (soma dos dias de pastejo e de descanso do pasto). Para simular o crescimento do pasto, foi utilizada a equação de Verhulst. Simulações efetuadas em um modelo computacional formulado no aplicativo Berkeley Madonna indicaram que, quando a entrada dos animais no pasto foi mais precoce (até os 73 dias), o sistema rotativo proporcionou um maior consumo de matéria seca pelos animais que o sistema de pastejo contínuo. Já quando a entrada dos animais no pasto foi entre os 74 e os 95 dias, o sistema contínuo proporcionou um maior consumo de matéria seca pelos animais que o sistema de pastejo rotativo. As simulações indicaram também que, quando o pastejo rotativo é iniciado precocemente (60 dias), o ciclo de 30 dias (um dia de pastejo e 29 de descanso) proporcionou maior consumo de matéria seca pelos animais. Já quando o pastejo rotativo é iniciado tardiamente (75 dias), variações da extensão do ciclo de rotação tiveram pouca influência sobre o consumo de matéria seca pelos animais.

Palavras-chave: simulação de sistemas dinâmicos, produção forrageira, modelo de Verhulst.

\section{ABSTRACT}

The main objective of the paper is to propose a dynamic systems simulation model for the analysis of pasture management seeking to maximize animal intake. The forage used was annual ryegrass, grazed by bovine adults. The variables considered were: animal stocking density, forage growth rate, animal apprehension rate, first entrance of the animals in the pasture (beginning of grazing) and rotation cycle (period of grazing plus period of rest of the pasture). To simulate the pasture growth the Verhulst equation was used. The computational model was programmed using software Berkeley Madonna. Simulations with this software showed that when grazing started early (until the 73 days) the rotative system provided greater animal dry matter intake than the continuous grazing system. When the grazing started between 74 and 95 days the continuous system provided greater animal dry matter intake than the rotative system. The simulations also showed that when the rotative system started early (60 days), the cycle of 30 days (a day of grazing and 29 of rest) provided greater animal dry matter intake. When the rotative system started later (75 days), variations of the grazing cycle length had little influence on dry matter intake.

Key words: dynamic systems simulation, forage production, Verhulst model.

\section{INTRODUÇÃO}

A simulação de sistemas dinâmicos pode ser usada para diminuir as dificuldades encontradas para analisar problemas em que observações diretas podem ser demoradas ou de custo muito elevado (HANNON \& RUTH, 1997). O manejo rotativo de pastagens pode ser considerado como um caso característico desse tipo de problema. Mesmo considerando apenas as combinações entre diferentes épocas de início do pastejo e períodos de utilização e

\footnotetext{
${ }^{1}$ Departamento de Estudos Agrários da Universidade Regional do Noroeste do Estado do Rio Grande do Sul (UNÍJUI). Rua São Francisco, 501, 98700-000, Ijuí, RS, Brasil. E-mail: bsneto@unijui.tche.br. Autor para correspondência.

${ }^{2}$ Faculdade de Itapiranga (FAI), Rua Estrela, 222, 89897-000, São João do Oeste, SC, Brasil. E-mail: marischneider2003@yahoo.com.br.

${ }^{3}$ Departamento de Zootecnia, Universidade Federal de Santa Maria (UFSM). Cidade Universitária, s/n, Camobi, 97105-900, Santa Maria, RS, Brasil. E-mail: jviegas@smail.ufsm.br.
} 
de descanso do pasto, a avaliação experimental do conjunto das alternativas possíveis envolveria custos extremamente elevados. Tal custo pode ser reduzido significativamente pela aplicação de técnicas de modelagem e pela simulação de sistemas dinâmicos, as quais se encontram disponíveis atualmente em vários pacotes computacionais comerciais confiáveis e de grande capacidade. Tais técnicas podem ser utilizadas, por exemplo, para definir as combinações potencialmente mais produtivas, as quais poderiam então ser validadas experimentalmente.

Nosso interesse é mostrar como a simulação de sistemas dinâmicos pode ser usada para analisar o manejo de pastagens. Este trabalho tem como objetivo principal propor um modelo de simulação de sistemas dinâmicos, formulado no software Berkeley Madonna (HANNON \& RUTH, 1997), para analisar os sistemas de pastejo rotativo e contínuo. Para a construção do modelo, foi utilizada a equação de Verhulst, considerada como uma das mais realísticas do ponto de vista biológico (BASSANEZI, 2002). A forrageira utilizada nas simulações foi o azevém anual, sendo o seu manejo direcionado para maximizar o consumo de matéria seca por bovinos adultos. A escolha dessa forrageira se deu por ela ser bastante conhecida e usada na alimentação de bovinos no Rio Grande do Sul, tanto na produção de leite como na produção de carne.

\section{MATERIAL E MÉTODOS}

Neste trabalho, a equação de Verhulst foi utilizada para a estimativa do crescimento do azevém anual. As características da equação de Verhulst estão relacionadas à taxa potencial de crescimento $(\mathrm{k})$ e ao valor máximo da variável que pode ser atingido pela variável estimada (Pmáx) (BASSANEZI \& FERREIRA JR, 1998). Esse modelo pode ser descrito como:

$$
\frac{d M}{d t}=k M\left(1-\frac{M}{M_{\text {máx }}}\right) \quad \mathrm{k}>0
$$

onde:

$$
\begin{aligned}
& \frac{d M}{d t}=\text { acumulação por unidade de tempo; } \\
& k=\text { taxa potencial de crescimento; } \\
& M=\text { variável estimada; } \\
& M_{\text {máx }}=\text { limite máximo da variável estimada; } \\
& t=\text { tempo. }
\end{aligned}
$$

Na figura 1, é mostrada a curva de acúmulo de matéria seca obtida pelo ajuste da equação de Verhulst a dados de crescimento de azevém comum obtidos na Estação Experimental da Universidade de Santa Maria por Quadros (2004, informação pessoal). No ajustamento dessa curva, os valores dos coeficientes da equação, a soma dos quadrados dos desvios e os coeficientes de correlação obtidos por meio da minimização da soma dos quadrados dos desvios foram:

- Taxa potencial de crescimento $(\mathrm{k})=0,04769\left(\mathrm{dia}^{-1}\right)$;

- Matéria seca máxima $\left(\mathrm{M}_{\text {máx }}\right)=7465$ (kg matéria seca $\left.\mathrm{ha}^{-1}\right)$;

- Soma dos quadrados dos desvios = 363472;

- Coeficiente de correlação = 0,995.

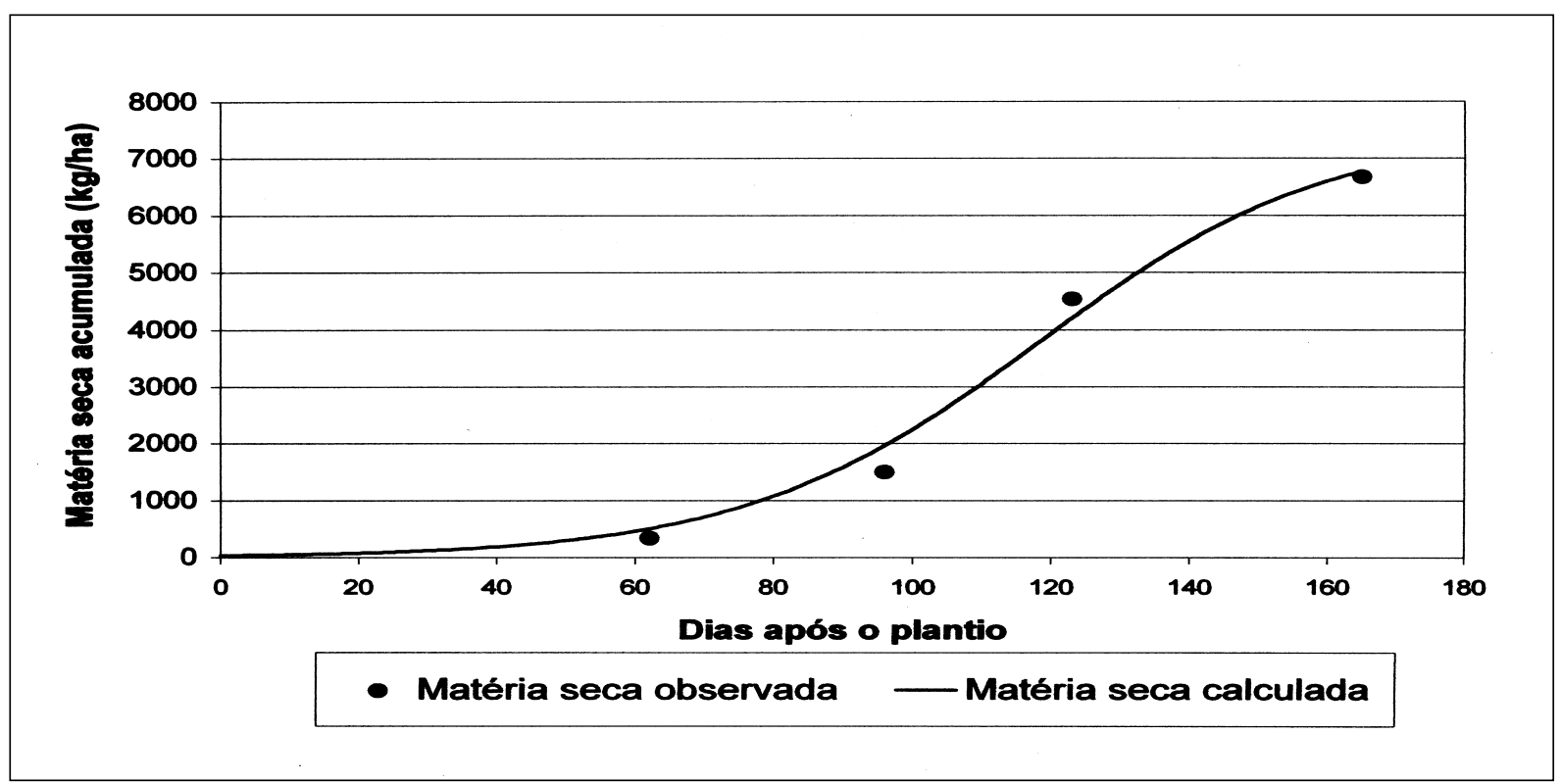

Figura 1 - Matéria seca em pasto de azevém anual observada e estimada pelo ajuste da equação de Verhulst.

Ciência Rural, v.36, n.4, jul-ago, 2006. 
O coeficiente de correlação obtido foi elevado e o ajuste da equação resultou também em um máximo de matéria seca compatível com os valores de matéria seca total acumulada pelo azevém anual indicados pela literatura (ALCÂNTARA \& BUFARAH, 1982).

O modelo de simulação

De acordo com procedimento descrito por WOODWARD \& WAKE (1995), o modelo utilizado neste trabalho foi formulado de forma que, quando o piquete estiver sob pastejo, a matéria seca consumida pelos animais será subtraída do crescimento do pasto, o qual é proporcional à própria matéria seca acumulada. Quando o piquete estiver em descanso, o crescimento do pasto ocorre segundo a equação de Verhulst, cujo ajuste foi descrito acima.

A taxa diária de consumo de matéria seca pelos animais pode ser descrita como

$\frac{d c}{d t}=A n M$

onde:

$\frac{d c}{d t}=$ consumo diário de matéria seca (kg matéria seca

ha' ${ }^{-1}$ dia $\left.^{-1}\right)$;

$n=$ número de animais;

$A=$ taxa da capacidade de apreensão de um animal (animal $^{-1}$ dia $^{-1}$ ) $\quad 0 \leq \mathrm{A}<1$;

$M=$ matéria seca acumulada (kg matéria seca ha-1).

A equação que representa a taxa diária de consumo de matéria seca dos animais é acrescentada à equação (1) para dar a acumulação líquida:

$$
\frac{d M}{d t}=k M\left(1-\frac{M}{M_{\text {máx }}}\right)-A n M
$$

onde:

$$
\frac{d M}{d t}=\text { acumulação diária líquida de matéria seca (kg }
$$

matéria seca ha-1 dia $^{-1}$ );

$M_{\text {máx }}=$ matéria seca máxima ( $\mathrm{kg}$ matéria seca ha $\left.{ }^{-1}\right)$;

$k=$ taxa potencial de crescimento $\left(\mathrm{dia}^{-1}\right)$.

O manejo dos animais entre os diversos piquetes foi definido a partir das variáveis dias de descanso (D) e dias de pastejo (P). Nos períodos de descanso (D), a equação (3) é aplicada com o número de animais (n) igual a zero. Cada ciclo (C) representa a soma dos períodos de pastejo e de descanso de cada piquete, ou seja, $\mathrm{C}=\mathrm{P}+\mathrm{D}$, sendo o número de piquetes definido por $(\mathrm{D} / \mathrm{P})+1$. A rotação entre os piquetes foi formulada por meio de funções lógicas ("se... então..., se não...”), o que torna o modelo discreto, sendo por isso escolhido o método de integração de Euler (SANTOS et al., 2002).

O software Berkeley Madonna (HANNON \& RUTH, 1997) foi utilizado para formular o modelo desse trabalho, cujo código encontra-se descrito abaixo:

\{MODELO DE SIMULAÇÃO DE PASTEJO ROTATIVODE PASTAGENS

STARTTIME $=0$
STOPTIME $=150$
DT $=1$
METHODEULER

$\mathrm{S}=30$

$\mathrm{M}=7465$

$\mathrm{T}=0.05$

animais $=3$

$\mathrm{A}=0.2$

$\mathrm{CI}=15$

minms $=350$

$\mathrm{NP}=31$

$\mathrm{E}=75$

$\mathrm{D}=29$

$\mathrm{P}=1$

Ciclo $=\mathrm{D}+\mathrm{P}$

Piquetes $=\mathrm{D} / \mathrm{P}+1$

mínimo $=$ minms $/$ Piquetes

VPiquete[1..NP] = Piquete[i]

VTotal_Consumido[1..NP] = Total_Consumido[i]

VTCA[1..NP $]=$ TCA $[\mathrm{i}]$

VCP1[1..NP $]=\mathrm{CP}[\mathrm{i}]$

MST = ARRAYSUM(Piquete[*])

Disponivel_por_an_inicio = IF animais $>0$ THEN (IF

TIME=E THEN Piquete[1]/animais ELSE 0) ELSE 0

Consumo_total = ARRAYSUM(Total_Consumido[*])

Consumo_por_animal $=$ IF animais $>0$ THEN

Consumo_total/animais ELSE 0

Taxa_de_consumo_por_animal = IF animais $>0$ THEN

ARRAYSUM(TCA[*])/animais ELSE 0

Crescimento_da_pastagem $=$ ARRAYSUM(CP $[*])$

\{Produção de forragem e consumo pelos animais em cada piquete $\}$

D/DT $($ Piquete[1..NP]) = CP[i] - TCA[i]

INIT Piquete[1..NP] = IF Piquetes $<$ (i) THEN 0 ELSE S/ Piquetes

$\mathrm{CP}[1 . . \mathrm{NP}]=\mathrm{IF}$ Piquetes $<(\mathrm{i})$ THEN 0 ELSE Piquete[i]*T*(1-Piquete[i]/(M/Piquetes))

TCA[1..NP ] $=$ IF TIME $<$ E $+(\mathrm{i}-1) *$ P OR Piquetes $<$ (i) OR 
(Piquete[i] < mínimo) OR (MOD(TIME - (E+(i-1)*P), $\mathrm{P}+\mathrm{D})>=\mathrm{P})$

THENO

ELSE IF (A*Piquete[i]*animais) >=CI*animais

THEN CI*animais

ELSE A*Piquete[i]*animais

\{Total Consumido em cada piquete $\}$

D/DT (Total_Consumido[1..NP]) = TCA[i]

INIT Total_Consumido[1..NP] $=0$

onde:

S = matéria seca na emergência do pasto (considerada igual ao peso das sementes, $\mathrm{kg} \mathrm{ha}^{-1}$ );

$\mathrm{T}=$ taxa potencial de crescimento do pasto $\left(\mathrm{dia}^{-1}\right)$;

$\mathrm{M}=$ limite máximo de matéria seca do pasto $\left(\mathrm{kg} \mathrm{ha}^{-1}\right)$;

$\mathrm{E}=$ primeira entrada dos animais na pastagem (dias);

minsm = quantidade mínima de matéria seca que os animais conseguem pastar $\left(\mathrm{kg} \mathrm{ha}^{-1}\right)$;

$\mathrm{NP}=$ número máximo de piquetes (unidades);

$\mathrm{P}=$ período de pastejo (dias);

$\mathrm{D}=$ período de descanso (dias);

A = capacidade de apreensão de matéria seca pelos animais (animal $\left.{ }^{-1} \mathrm{dia}^{-1}\right)$;

animais = lotação (número de animais ha-1);

CI = capacidade de ingestão de matéria seca por animal $\left(\mathrm{kg}\right.$ animal $\left.{ }^{-1}\right)$;

Piquetes $=$ número total de piquetes (unidades);

Piquete[i] = matéria seca acumulada no piquete i $(\mathrm{kg}$ ha $^{-1}$ );

$\mathrm{CP}[\mathrm{i}]$ = aumento de matéria seca no piquete i (equação de Verhulst, $\mathrm{kg} \mathrm{ha}^{-1} \mathrm{dia}^{-1}$ );

TCA[i] = consumo de matéria seca pelos animais no piquete i $\left(\mathrm{kg} \mathrm{ha}^{-1} \mathrm{dia}^{-1}\right)$.

Assim, as variáveis independentes do modelo são: lotação animal, época da primeira entrada dos animais na pastagem, ciclo de rotação (períodos de pastejo e de descanso dos piquetes), taxa potencial de crescimento do pasto, limite máximo de matéria seca do pasto, capacidade de apreensão diária de forragem pelos animais e capacidade de ingestão de matéria seca pelos animais. As variáveis dependentes são: número de piquetes, produção consumida e residual de cada piquete e consumo total e por animal de matéria seca. Neste trabalho, foram efetuadas apenas simulações relativas à época de entrada dos animais na pastagem, ao ciclo de rotação e à comparação entre o pastejo rotativo e o contínuo (sendo esse considerado um caso limite, em que o período de descanso é nulo).

A parametrização do modelo

Por meio de entrevistas com alguns agricultores da região de Ijuí e com técnicos da
Cooperativa Tritícola Serrana (COTRIJUÍ), constatouse que o azevém, em geral, é semeado em abril e pastejado a partir do momento em que disponibiliza 1500 a $1700 \mathrm{~kg}$ de matéria seca ha ${ }^{-1}$. Além disso, de acordo com as informações obtidas, foi considerada uma lotação (n) de 3 vacas ha-1, sendo a taxa de apreensão diária (A) por animal fixada em 20\% da matéria seca do pasto, para que os animais possam consumir em torno de $60 \%$ do pasto. Foi considerado que, pela forma de apreensão dos bovinos, esses não conseguem pastar forragens com menos de 5 centímetros de altura (350 $\mathrm{kg} \mathrm{ha}^{-1}$ de matéria seca). A taxa potencial de crescimento do pasto ajustada foi aproximada para $5 \%(k=0,05)$ e a produção máxima foi considerada de $7465 \mathrm{~kg}$ de matéria seca ha ${ }^{-1}$. Já a capacidade de ingestão (CI) dos animais foi fixada num limite máximo de $15 \mathrm{~kg}$ de matéria seca animal ${ }^{-1} \mathrm{dia}^{-1}$, ou seja, 3\% do seu peso vivo. Foi considerada uma área de 1 hectare, onde foram semeados $30 \mathrm{~kg}$ de sementes de azevém anual, sendo que essa área pode ser dividida em até 31 piquetes. O período total de crescimento do pasto (da emergência até o final do ciclo) foi fixado em 150 dias.

\section{RESULTADOS E DISCUSSÃO}

Uma série de simulações foi realizada para verificar a influência do ciclo de rotação (dias de pastejo mais dias de descanso) sobre a matéria seca consumida pelos animais, considerando duas épocas de entrada dos animais na pastagem (75 e 60 dias após a emergência). Nessas simulações, o período de pastejo foi fixado em 1 dia, variando-se o período de descanso. Os resultados dessas simulações estão apresentados na figura 2, os quais mostram que, no ciclo de 18 dias (1 de pastejo mais 17 de descanso), a diferença no total consumido entre as épocas de entrada foi elevada (cerca de 832 $\mathrm{kg} \mathrm{ha}^{-1}$ ). Porém, na medida em que o ciclo se torna maior, essa diferença diminui, chegando a $205 \mathrm{~kg}$ ha $^{-1}$ no ciclo de 31 dias, sendo a entrada aos 75 dias sempre superior.

Na entrada aos 75 dias, o melhor ciclo observado foi o de 23 dias (1 dia de pastejo e 22 dias de descanso), embora a influência do ciclo sobre o total consumido, nesse caso, tenha sido pequena, sendo o máximo de 3093 $\mathrm{kg} \mathrm{ha}^{-1}$, com um ciclo de 23 dias e o mínimo de 2982 $\mathrm{kg} \mathrm{ha}^{-1}$, com um ciclo de 31 dias.

Já na entrada aos 60 dias, o melhor ciclo foi o de 30 dias (1 dia de pastejo e 29 dias de descanso), com um total consumido de $2781 \mathrm{~kg} \mathrm{ha}^{-1}$ de matéria seca. Observa-se que, de maneira geral, o consumo aumenta a taxas decrescentes com o aumento do ciclo de rotação, 


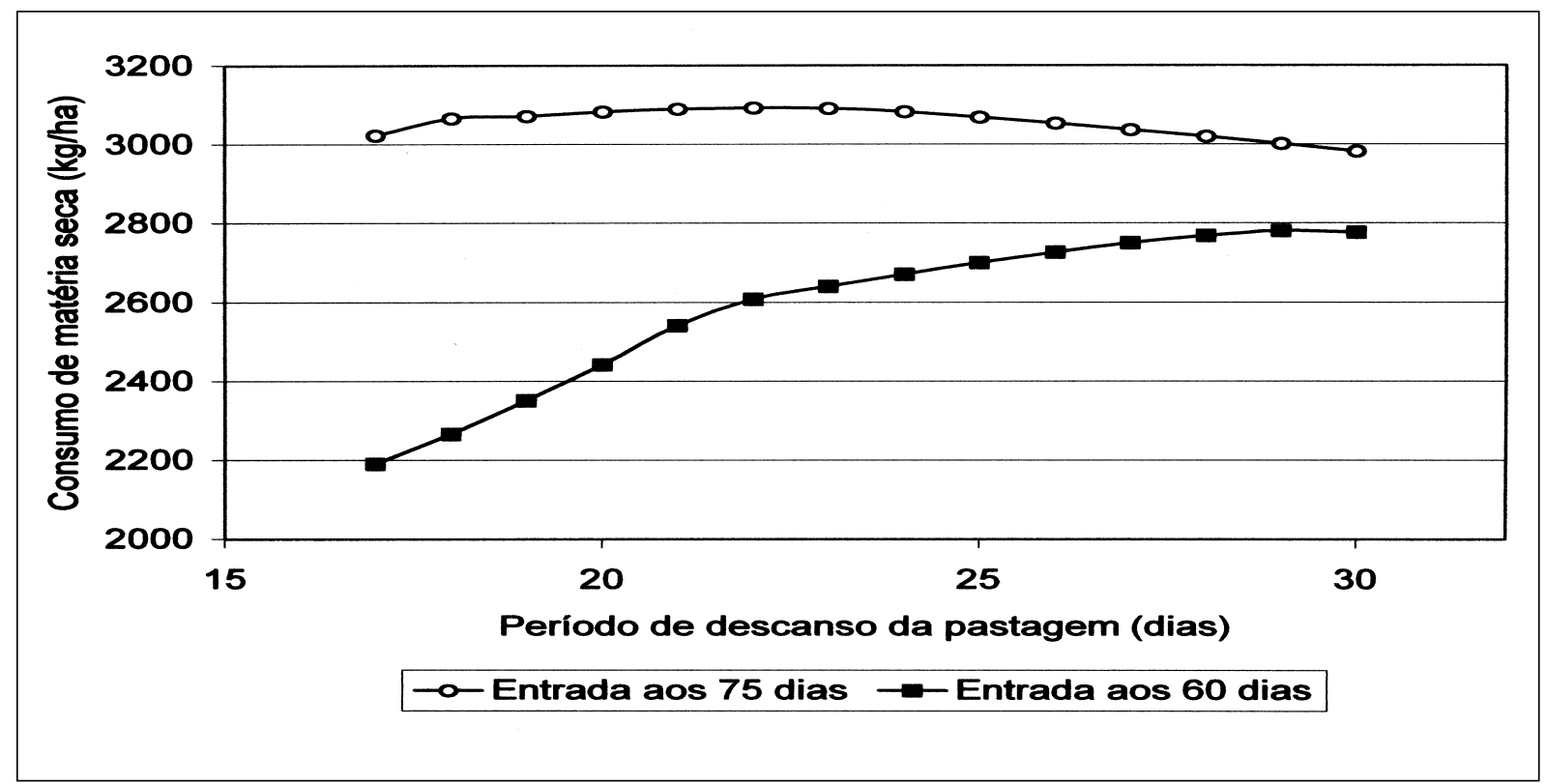

Figura 2 - Relação entre o ciclo de rotação e consumo de matéria seca até os 150 dias após a implantação do pasto, considerando duas épocas de início do pastejo.

com o crescimento tornando-se nulo a partir do ciclo de 30 dias.

Em suma, as simulações mostradas na figura 2 indicam que o sistema de pastejo rotativo com 30 piquetes, com 1 dia de pastejo e 29 de descanso (1+29), é um sistema interessante para o manejo do azevém para a bovinocultura. Segundo os técnicos da COTRIJUÍ consultados para a parametrização do modelo, esse ciclo (1+29) é o mais utilizado na região de Ijuí.

Uma segunda série de simulações foi realizada para analisar a influência da época de entrada na pastagem sobre o consumo total de matéria seca, nos sistemas de pastejo contínuo e rotativo. Neste último, foi adotado um ciclo de 30 dias, sendo 1 de pastejo e 29 de descanso. Os resultados dessas simulações são mostrados na figura 3. Conforme podemos observar nessa figura, nos dois tipos de sistema, o consumo total máximo ocorre aos 74 dias no sistema rotativo e aos 75 dias no sistema contínuo. Nessa época, o sistema de pastejo contínuo proporcionou um consumo de matéria seca pelos animais superior ao sistema rotativo. Por outro lado, é importante salientar que, na entrada aos 75 dias, os dados obtidos nas simulações indicaram que a matéria seca total e residual do pasto é mais elevada no sistema rotativo (5811 $\mathrm{kg} \mathrm{ha}^{-1}$ de matéria seca total e $2809 \mathrm{~kg} \mathrm{ha}^{-1}$ de matéria seca residual, sendo, portanto, o consumo de $3002 \mathrm{~kg} \mathrm{ha}^{-1}$ de matéria seca) que no sistema de pastejo contínuo (3970 $\mathrm{kg} \mathrm{ha}^{-1}$ de matéria seca total e $595 \mathrm{~kg} \mathrm{ha}^{-1}$ de matéria residual, sendo, portanto, o consumo de $3375 \mathrm{~kg} \mathrm{ha}^{-1}$ de matéria seca), apesar de o consumo de matéria seca ser menor.

No caso de a entrada ser antes dos 74 dias, o pastejo rotativo é superior, sendo o pastejo contínuo superior dos 74 dias até os 95 dias de entrada. Para épocas de entrada maiores que 95 dias, os dois sistemas proporcionam um consumo muito semelhante.

Em suma, as simulações efetuadas neste trabalho indicam que:

- há pouca influência do ciclo de rotação (dias de uso mais dias de descanso) sobre o consumo total, quando a entrada na pastagem é tardia (75 dias após a emergência);

- ciclos de pastejo mais longos (30 dias) proporcionaram consumos totais superiores, quando a entrada dos animais na pastagem foi precoce (60 dias);

- a utilização de uma entrada dos animais na pastagem mais tardia (75 dias) proporcionou um consumo total maior de matéria seca em relação ao consumo total proporcionado pela entrada mais precoce (60 dias); - quando a entrada dos animais na pastagem foi mais precoce (até os 73 dias), o sistema rotativo proporcionou um maior consumo de matéria seca pelos animais que o sistema de pastejo contínuo;

- quando a entrada dos animais na pastagem foi entre os 74 e os 95 dias, o sistema contínuo proporcionou um maior consumo de matéria seca pelos animais que o sistema de pastejo rotativo;

- para épocas de entrada dos animais na pastagem muito tardias (após 95 dias), os sistemas rotativo e contínuo 


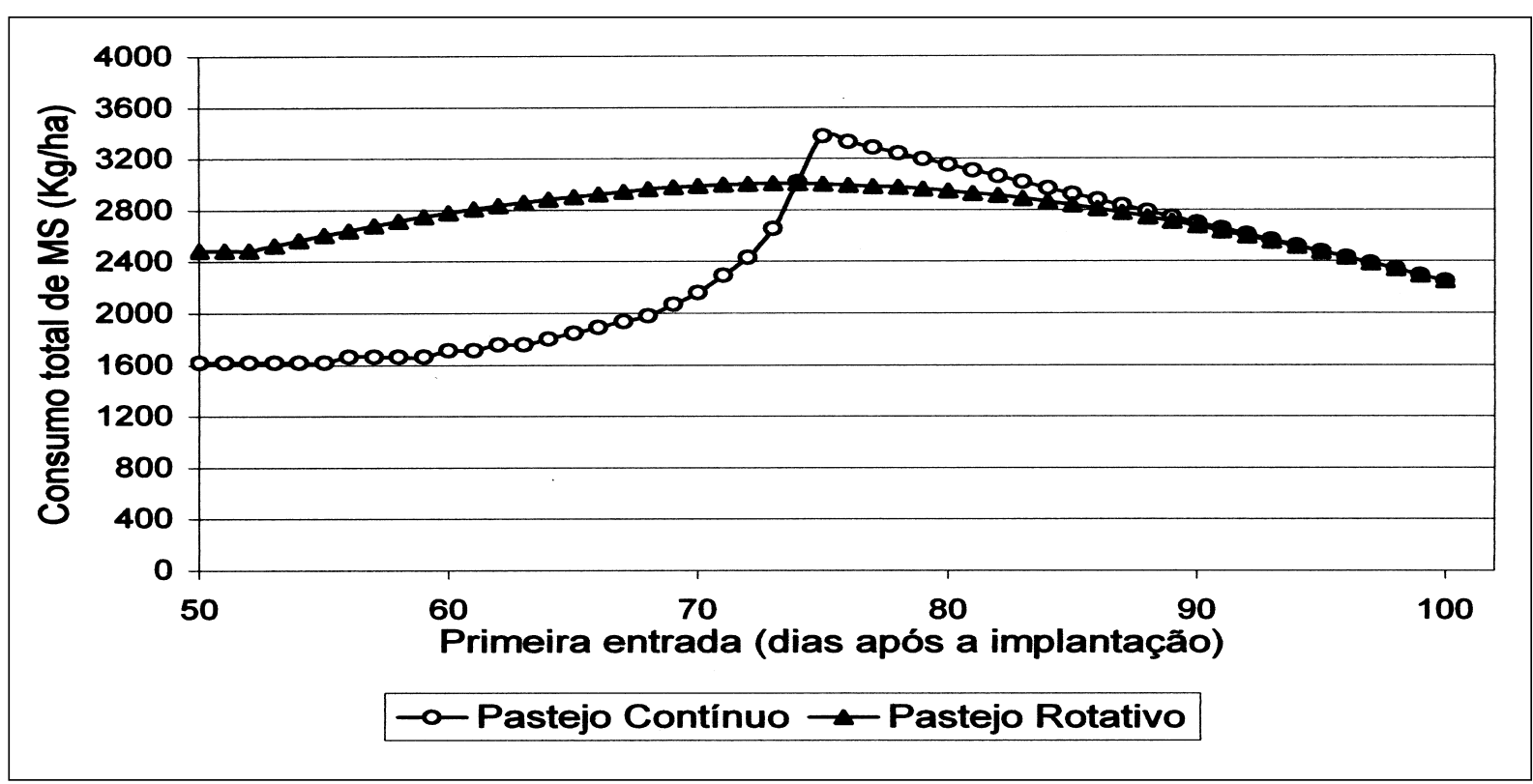

Figura 3 - Simulação do consumo total de azevém anual até 150 dias após a sua implantação em função da primeira entrada na pastagem.

proporcionaram consumos de matéria seca pelos animais semelhantes;

- o sistema rotativo, mesmo quando proporcionou um consumo menor pelos animais, proporcionou um acúmulo maior de matéria seca total e residual pela pastagem.

Enfim, é importante salientar o caráter exploratório deste trabalho, recomendando-se que os resultados obtidos, especialmente no que diz respeito ao manejo do azevém, sejam considerados com cautela.

\section{CONCLUSÕES}

Os resultados obtidos nesse trabalho indicam que o modelo desenvolvido permite a simulação da influência da época de entrada dos animais na pastagem e das características do ciclo de rotação sobre a produção, o consumo de matéria seca pelos animais e a quantidade residual desta última após a utilização do pasto.

\section{INFORMAÇÕES VERBAIS}

As informações verbais contidas neste artigo foram obtidas junto ao Prof. Fernando Quadros, do Departamento de
Zootecnia da Universidade Federal de Santa Maria, Faixa de Camobi, Km 9, Campus Universitário Santa Maria, 97105900, Santa Maria - RS, e-mail: fquadros@ccr.ufsm.br.

\section{REFERÊNCIAS}

ALCÂNTARA, P. B.; BUFARAH, G. Plantas forrageiras: gramíneas e leguminosas. São Paulo: Nobel, 1982. 150 p.

BASSANEZI, R.C.; FERREIRA JR., W.C. Equações diferenciais com aplicações. São Paulo: HARBRA, 1998. 572 p.

BASSANEZI, R.C. Ensino-aprendizagem com modelagem matemática. São Paulo: Contexto, 2002. 389p.

HANNON, B.; RUTH, M. Modeling dynamic biological systems. New York: Springer - Verlag, 1997. 399p.

SANTOS, A. de C.K. dos; et al. Modelagem computacional utilizando STELLA: considerações teóricas e aplicações em gerenciamento, física e ecologia de sistemas. Rio Grande: FURG, 2002. 138p.

WOODWARD, S.J.R.; WAKE, G.C. Optimal grazing of a multipaddock system using a discrete time model. Agricultural Systems, Oxford, n.48, p.119-139, 1995. 